\title{
Statin and metformin therapy in prostate cancer patients with hyperlipidemia who underwent radiotherapy: a population-based cohort study
}

This article was published in the following Dove Medical Press journal: Cancer Management and Research

Ke Li, I,* Jie Si-Tu, I,* Jianguang Qiu, ${ }^{2, *} \mathrm{Li} \mathrm{Lu},{ }^{2, *}$ Yunhua Mao,' Hua Zeng, ${ }^{3}$ Mingkun Chen, ${ }^{4}$ Caiyong Lai, ${ }^{5}$ Heng-Jui Chang, ${ }^{6}$ Dejuan Wang ${ }^{2}$

'Department of Urology, The Third Affiliated Hospital of Sun Yat-sen University, Guangzhou, Guangdong Province 510630, China; ${ }^{2}$ Department of Urology, The Sixth Affiliated Hospital of Sun Yat-sen University, Guangzhou, Guangdong Province 510655, China; ${ }^{3}$ Department of Emergency, The Third Affiliated Hospital of Sun Yat-sen University, Guangzhou, Guangdong Province 510630, China; ${ }^{4}$ Department of Urology, The Third Affiliated Hospital of Southern Medical University, Guangzhou, Guangdong Province 51 0630, China; ${ }^{5}$ Department of Urology, The First Affiliated Hospital of Jinan University, Guangzhou, Guangdong Province 510632, China; ${ }^{6}$ Department of Radiation Therapy Oncology, Min-Sheng General Hospital, Taoyuan 330, Taiwan

*These authors contributed equally to this work

Correspondence: Dejuan Wang

Department of Urology, The Sixth

Affiliated Hospital of Sun Yat-sen

University, Yuancun Erheng Road 26,

Guangzhou 510655, China

Tel +862038379697

Fax +86 2038254220

Email wdj_sysu@I26.com

Heng-Jui Chang

Department of Radiation Therapy Oncology, Min-Sheng General Hospital, 168, ChingKuo Rd, 330 Taoyuan, Taiwan Tel +88633I79599

Email bryancgu@yahoo.com.tw
Purpose: To evaluate the association between the use of statins and/or metformin and patient survival in prostate cancer patients in Taiwan.

Subjects and methods: Newly diagnosed prostate cancer patients who had hyperlipidemia and received radiotherapy were identified from the National Health Insurance Research Database 2000-2010. The survival rate was estimated by the Kaplan-Meier method. Univariate and multivariate Cox regression analyses were performed to examine the association of mortality. Sensitivity analysis was performed to assess the risk of mortality in patients with diabetes.

Results: The study included 567 patients. Patients who used statins or metformin after prostate cancer diagnosis had longer average survival times (9.3 years and 8.1 years, respectively; $P=0.001$ ) compared with patients who persistently used or used the medicines prior to cancer diagnosis. Multivariate Cox regression analysis found that patients treated with statins after cancer diagnosis were significantly associated with a lower risk of mortality (aHR $=0.24,95 \%$ $\mathrm{CI}=0.09-0.66$ ) compared to patients who did not use statins during the study period. Patients treated with metformin after cancer diagnosis were significantly associated more with an increased risk of mortality ( $\mathrm{aHR}=6.78,95 \% \mathrm{CI}=2.45-18.77$ ) compared to patients who did not use metformin during the study period. Sensitivity analysis revealed that the average survival time was similar among different medicine use groups in patients with diabetes.

Conclusion: The finding suggests that statins and metformin use after prostate cancer diagnosis may increase survival in patients with hyperlipidemia and radiotherapy.

Keywords: prostate cancer, metformin, statin, hyperlipidemia, mortality, survival

\section{Introduction}

Cancer has been the leading cause of death in Taiwan for more than a decade. ${ }^{1}$ Prostate cancer is ranked the fifth most common cancer in Taiwan, with the seventh highest cancer-related mortality rate. ${ }^{2}$ The incidence of prostate cancer has been rapidly increasing in the past 10 years. $^{3}$

Findings from several retrospective observational studies indicate that treatment with statins or metformin can reduce prostate-specific antigen (PSA) levels. ${ }^{4-8}$ Statin use is found to be associated with a lower advanced prostate cancer risk and reduced prostate cancer-specific mortality. ${ }^{9}$ Statins are potent inhibitors of 3-hydroxy-3-methylglutaryl coenzyme A reductase, and are commonly used for treating hyperlipidemia by lowering cholesterol., ${ }^{9,10}$ Cholesterol plays a role in steroid androgens that drive prostate growth. ${ }^{11}$ In addition, rapidly growing tumor cells also require high levels of cholesterol. A number of reports suggest that statins are associated with a reduc- 
tion in the incidence of prostate cancer, disease recurrence, and better overall survival in patients; however, others have found no such benefits with statin therapy. ${ }^{12-17}$ However, a link between cholesterol and reduction of PSA is not clear. ${ }^{4,5}$

Metformin is a commonly used antidiabetic medication and is an insulin-sensitizing oral biguanide. Several in vitro studies suggest that the use of metformin may be protective against prostate cancer or delay disease progression. ${ }^{18-21}$ Prior studies suggest that metformin may show a benefit as adjunctive therapy to standard prostate cancer therapy, particularly in patients receiving androgen deprivation therapy (ADT). ${ }^{22,23}$ However, for metastatic castration-resistant prostate cancer, it has been suggested that metformin may not be an effective chemosensitizer. ${ }^{24}$ Furthermore, several studies have found that metformin did not improve biochemical-free or cancerspecific survival in patients treated with permanent interstitial brachytherapy. ${ }^{25,26}$ Deng et al ${ }^{27}$ suggested that there was no association between the metformin treatment and all-cause mortality or recurrence, but yet metformin therapy may decrease the incidence of prostate cancer.

The results for use of statins and/or metformin in prostate cancer were controversial and should be confirmed with further investigation. Statins and metformin are commonly used for treating hyperlipidemia. ${ }^{10,28}$ Studies exploring the association between the use of statins and/or metformin and patient survival in prostate cancer patients in Taiwan have never been reported. Thus, the current study aimed to assess associations of statins and/or metformin therapy and survival of prostate cancer patients with hyperlipidemia and radiotherapy in Taiwan. The study was a retrospective, nationwide, Taiwanese-based study using the database from the Taiwan National Health Insurance..$^{29,30}$

\section{Subjects and methods Data source}

Taiwan launched a single-payer National Health Insurance program on March 1, 1995. ${ }^{29}$ The database of this program, called The National Health Insurance Research Database (NHIRD), contains registration files and original claim data for reimbursement. The NHIRD represents a comprehensive nationwide database that includes information since 1995 and which covers $99 \%$ of Taiwan's 23.74 million people, $98 \%$ of whom are Han Chinese. The database is maintained by the National Health Research Institutes in Taiwan and provided to scientists in Taiwan for research purposes. The NHIRD databases provide excellent data sources for population-level analysis, with minimal discrepancies and biases. ${ }^{31}$
This study was approval by the Institutional Review Board of the Third Affiliated Hospital of Sun Yat-sen University. Given the retrospective nature of this study design, the requirement for obtaining informed consent was waived.

\section{Study population}

A total of 5,079 males newly diagnosed with prostate cancer were identified from the NHIRD database during 2000-2010 using the appropriate International Statistical Classification of Diseases code (ICD9=185). After excluding 3,020 patients who did not have hyperlipidemia (ICD9=272) and 1,492 patients who did not receive radiotherapy after being diagnosed with prostate cancer, only 567 patients were included in this study (Figure 1).

\section{Variable definitions}

The primary outcome was overall survival. Independent variables included demographic characteristics (age), the use of medicines, such as statin (ATC code: C10AA01C10AA05, C10AA07, and C10AA08) or/and metformin (ATC code: A10BA02), and the comorbidities, such as obesity (ICD-9-CM 278.0), diabetes mellitus (ICD-9-CM 250), and metabolic syndrome (ICD-9-CM 277.7). Furthermore, subjects with the use of statin only and metformin only were subdivided into three groups: the pre-diagnostic use group (use of statin or metformin prior to prostate cancer diagnosis), the post-diagnostic use group (use of statin or metformin after diagnosis of prostate cancer), and the persistent use group (continuous use of the medicines before and after the cancer diagnosis).

\section{Statistical analysis}

The continuous variable was expressed as the mean and SD. Categorical variables were expressed as counts and percentages. Kaplan-Meier survival analysis was performed for medicine use (ie, statin and/or metformin) with the endpoint of the mortality, and the statistical significance was examined using a log-rank test. In survival analysis, any cause of deaths was treated as events and survivors were treated as censored events. Univariate and multivariate Cox proportional hazards regression analyses were performed to examine the association of mortality. The multivariate Cox proportional hazards regression model was simultaneously adjusted for the baseline characteristics, including age, diabetes mellitus, hypertension, cardiovascular disease, peripheral artery disease, and atherosclerosis. Furthermore, subgroup analyses were performed for patients using statin only or metformin 
only to examine the association of mortality. In addition, a sensitivity analysis was carried out to assess the association of mortality in patients with diabetes.

All statistic assessments were two-sided and evaluated at the 0.05 level of significance. Statistical analyses were performed by IBM SPSS statistical software version 22 for Windows (IBM Corporation, Armonk, NY, USA).

\section{Results}

The average age of the study population was 71.8 years. A total of $213(37.6 \%)$ patients did not use either statin or metformin, 121 (21.3\%) took both drugs, 174 (30.7\%) only received the statin, and $59(10.4 \%)$ were only administered metformin. The most common comorbidities were diabetes

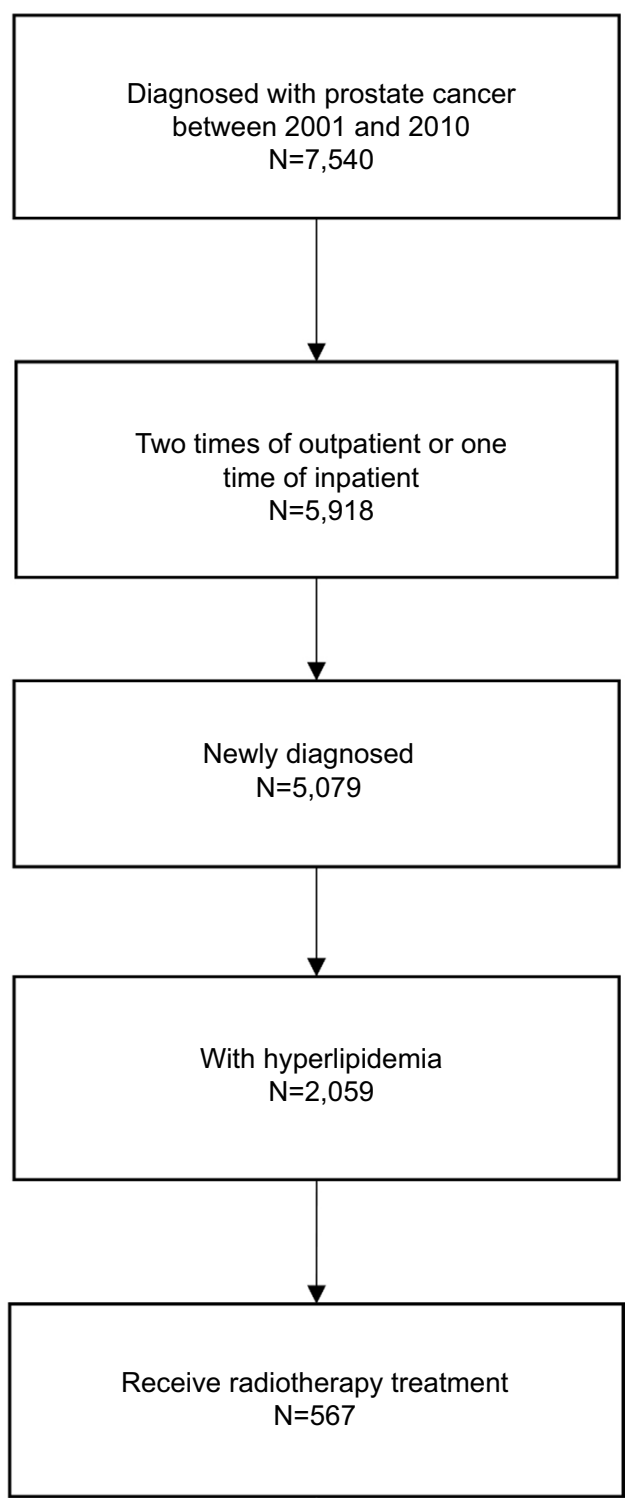

Figure I Flowchart of study population. mellitus (53.8\%), hypertension (77.7\%), and cardiovascular disease $(66.3 \%)$. During the follow-up period, 137 deaths $(24.2 \%)$ occurred in the total study population (Table 1$)$. A higher percentage of patients with diabetes mellitus used metformin $(88.1 \%, P<0.001)$ compared with those taking statins, a combination of metformin and statins, or did not use either drug (Table S1). A greater percentage of patients with hypertension took statins $(84.3 \%, P=0.046)$ compared with not using either drug or taking both drugs. A larger percentage of patients with cardiovascular disease used only statins $(73.0 \%, P=0.009)$ compared with the other evaluated treatment regimens.

Kaplan-Meier analysis found that the average survival time for the total study population was 7.5 years (Figure 2 and Table 2). The survival time was similar for patients who did not use statins or metformin (the unused group, 7.1 years), who used both statins and metformin (the both group, 7.8 years), who only received statins ( 7.8 years), or who only took metformin (6.9 years) (Log-rank test, $P=0.407$ ) (Figure 2). Multivariate Cox regression analysis indicated that, compared to the unused group, the other three groups (both, only use statins, and only use metformin) were associated with lower risk of mortality, although the differences were not significant (Table 2). Furthermore, subgroup analyses were performed in which patients were divided into three medicine use categories: persistent use, pre-diagnostic use, and postdiagnostic use. The result of Kaplan-Meier analysis showed that patients with post-diagnostic use of statin had a longer average survival time (9.3 years, Log-rank test, $P=0.001$ )

Table I Subject characteristics

\begin{tabular}{|l|l|l|}
\hline Variable & n/mean & $\% /$ SD \\
\hline Age (years) & 71.8 & 8.7 \\
Medicine use & & \\
Unused & 213 & 37.6 \\
Both & 121 & 21.3 \\
Only use statin & 174 & 30.7 \\
$\quad$ Persistent use & 74 & 42.5 \\
$\quad$ Pre-diagnostic use & 55 & 31.6 \\
$\quad$ Post-diagnostic use & 45 & 25.9 \\
Only use metformin & 59 & 10.4 \\
$\quad$ Persistent use & 35 & 59.3 \\
$\quad$ Pre-diagnostic use & 7 & 11.9 \\
$\quad$ Post-diagnostic use & 17 & 28.8 \\
Comorbidities & & \\
Diabetes mellitus & 305 & 53.8 \\
Hypertension & 437 & 77.1 \\
Cardiovascular disease & 376 & 66.3 \\
Peripheral artery disease & 41 & 7.2 \\
Atherosclerosis & 51 & 9.0 \\
Mortality & 137 & 24.2 \\
\hline
\end{tabular}


(Table 3 and Figure 3). A similar result was also observed in patients with post-diagnostic use of metformin (8.1 years, Log-rank test, $P<0.001$ ) (Table 3 and Figure 3 ).

Multivariate Cox regression analysis revealed that patients who were treated with statins after the diagnosis of prostate cancer were significantly associated with a lower risk of mortality $(\mathrm{aHR}=0.24,95 \% \mathrm{CI}=0.09-0.66, P<0.05)$ compared to patients who did not use statins during the study period (Table 3 ). In contrast, patients who were treated with metformin before prostate cancer diagnosis were significantly associated with a higher risk of mortality (aHR $=6.78,95 \%$ $\mathrm{CI}=2.45-18.77, P<0.05)$ compared to patients who did not use metformin during the study period (Table 3 ).

A sensitivity analysis was performed to evaluate the risk of mortality in patients with diabetes, and the results revealed that the average survival time was similar among different medicine use groups in diabetic patients (Log-rank test, $P=0.906$ ) (Table S2). As shown in Table S3, diabetic patients

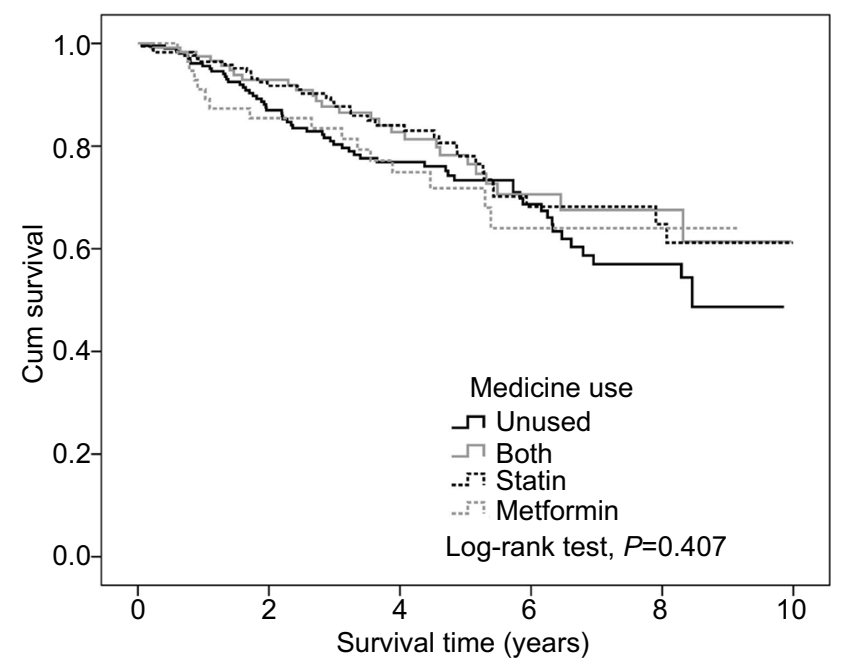

Figure 2 Kaplan-Meier analysis of differences in survival time among medicine use in total population. without statin use had a significantly longer average survival time ( 7.5 years, Log-rank test: $P=0.006)$. In contrast, diabetic patients with post-diagnostic use of metformin had significantly longer average survival time (7.9 years, Log-rank test: $P<0.001$, Table S3). Multivariate Cox analysis revealed that diabetic patients with persistent use and pre-diagnostic use of statins were significantly associated with a significantly higher risk of mortality $(\mathrm{aHR}=2.57$ and 2.96, respectively, Table S3) compared to those without statin therapy. Furthermore, diabetic patients with pre-diagnostic use of metformin were significantly associated with a higher risk of mortality compared to those without using metformin ( $\mathrm{aHR}=7.59$, $95 \%$ CI $=2.58-22.33, P<0.05$, Table S3).

\section{Discussion}

To the best of our knowledge, this is the first retrospective study that evaluated if the use of statins and/or metformin improved survival in Taiwanese prostate cancer patients who had hyperlipidemia and received radiotherapy. Use of statins or metformin, alone or in combination, did not improve survival time compared with patients who had received neither therapy. However, multivariate analysis found patients without these treatments were associated with higher risk of mortality, although the finding was not statistically significant. Patients who received statins after diagnosis of prostate cancer had a longer average survival time compared with patients who persistently used statins or had statin therapy prior to diagnosis. Similarly, patients who used statins after diagnosis were associated with a reduced risk of mortality compared to those who did not receive or persistently used statins. Patients who received metformin therapy after diagnosis of prostate cancer had a longer average survival time than patients who persistently used the drug or received metformin treatment after diagnosis. However, the use of metformin in patients before diagnosis was significantly

Table 2 Survival analysis of the medicine use in total population

\begin{tabular}{|c|c|c|c|c|c|c|}
\hline & $\begin{array}{l}n \text { of } \\
\text { events }\end{array}$ & $\begin{array}{l}\text { Mean for survival } \\
\text { time (years) }\end{array}$ & SE & $\begin{array}{l}\text { Log-rank test, } \\
P \text {-value }\end{array}$ & \begin{tabular}{|l|}
$\mathrm{HR}$ \\
$(95 \% \mathrm{Cl})$
\end{tabular} & $\begin{array}{l}\text { aHR } \\
(95 \% \mathrm{Cl})\end{array}$ \\
\hline Medicine use ${ }^{b}$ & 137 & 7.5 & 0.2 & 0.407 & & \\
\hline Unused $^{c}$ & 60 & 7.1 & 0.3 & & I & I \\
\hline Both $^{d}$ & 26 & 7.8 & 0.4 & & $0.75(0.47-1.19)$ & $0.68(0.42-1.09)$ \\
\hline Only use statin & 35 & 7.8 & 0.3 & & $0.74(0.49-1.12)$ & $0.77(0.50-1.19)$ \\
\hline Only use metformin & 16 & 6.9 & 0.5 & & $0.98(0.56-1.69)$ & $0.90(0.5 I-1.59)$ \\
\hline
\end{tabular}

Notes: Multivariate Cox proportional hazards regression model was simultaneously adjusted for age, diabetes mellitus, hypertension, cardiovascular disease, peripheral artery disease, and atherosclerosis. aln survival analysis, any cause of deaths was treated as events. bMedicine use was defined as using statin and/or metformin at any time

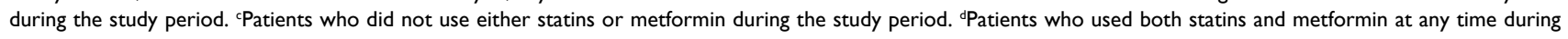
the study period.

Abbreviations: aHR, adjusted hazard ratio; SE, standard error. 
Table 3 Survival analysis of the medicine use in statin use only or metformin use only groups

\begin{tabular}{|c|c|c|c|c|c|c|}
\hline & $\begin{array}{l}n \text { of } \\
\text { events }\end{array}$ & $\begin{array}{l}\text { Average survival } \\
\text { time (years) }\end{array}$ & SE & $\begin{array}{l}\text { Log-rank } \\
\text { test, } P \text {-value }\end{array}$ & HR (95\% Cl) & aHR (95\% Cl) \\
\hline Only use statin & & & & $0.00 I^{b}$ & & \\
\hline Unused & 60 & 7.1 & 0.3 & & I & 1 \\
\hline Persistent use & 15 & 7.5 & 0.5 & & $0.78(0.44-1.37)$ & $0.78(0.43-I .4 I)$ \\
\hline Pre-diagnostic use & 16 & 5.3 & 0.5 & & $1.76(1.01-3.10)$ & $1.60(0.9 \mathrm{I}-2.82)$ \\
\hline Post-diagnostic use & 4 & 9.3 & 0.3 & & $0.22(0.08-0.60)$ & $0.24(0.09-0.66)$ \\
\hline Only use metformin & & & & $<0.00 \mathrm{I}^{\mathrm{b}}$ & & \\
\hline Unused & 60 & 7.1 & 0.3 & & 1 & 1 \\
\hline Persistent use & 8 & 7.1 & 0.6 & & $0.92(0.44-1.94)$ & $1.34(0.59-3.05)$ \\
\hline Pre-diagnostic use & 5 & 1.9 & 0.5 & & $6.37(2.5|-| 6.18)$ & $6.78(2.45-18.77)$ \\
\hline Post-diagnostic use & 3 & 8.1 & 0.5 & & $0.44(0.14-1.39)$ & $0.43(0.13-1.38)$ \\
\hline
\end{tabular}

Notes: Bold represented significant factor, $P<0.05$; multivariate Cox proportional hazards regression model was simultaneously adjusted for age, diabetes mellitus, hypertension, cardiovascular disease, peripheral artery disease, and atherosclerosis. aln survival analysis, any cause of deaths was treated as events. ${ }^{b}$ Represented significant difference of average survival time among the groups, log-rank test, $P<0.05$.

Abbreviations: aHR, adjusted hazard ratio, SE, standard error.

A

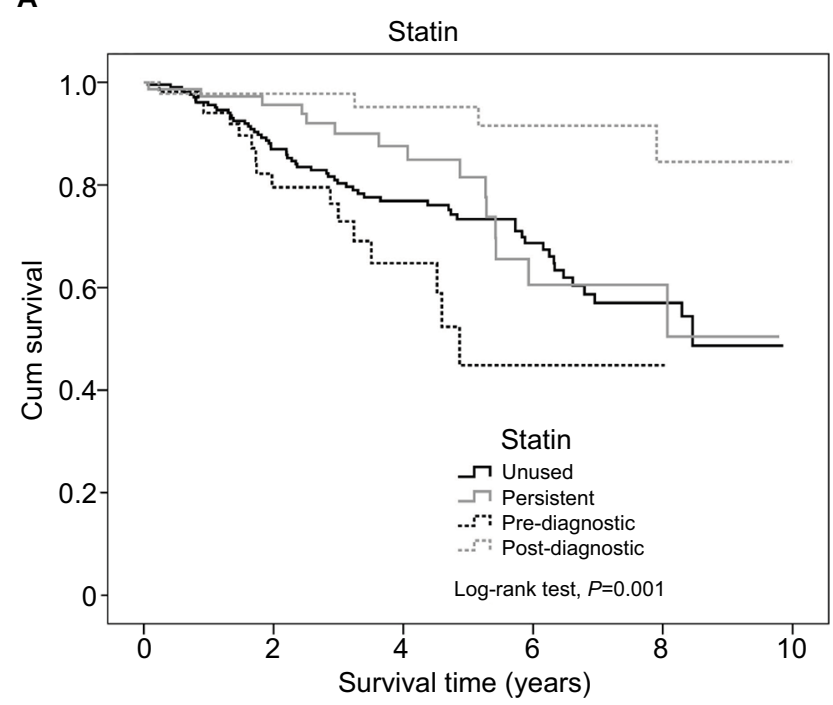

B

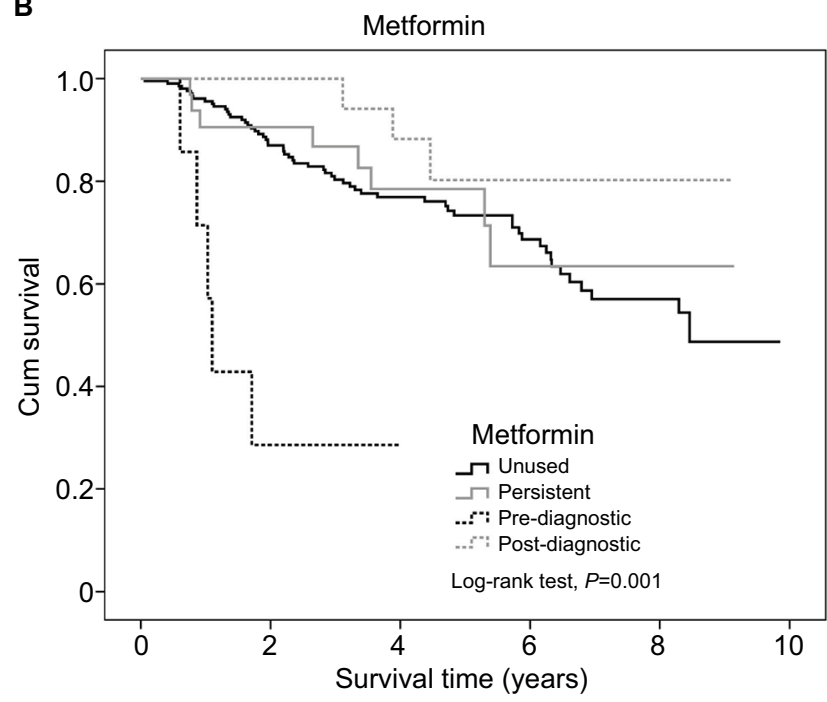

Figure 3 Kaplan-Meier analysis of differences in survival time between unused persistent, pre-diagnostic, and post-diagnostic use in (A) statin and (B) metformin. associated with a higher risk of mortality compared with patients who did not use metformin. The findings suggested that the use of statins and metformin in Taiwanese prostate cancer patients with hyperlipidemia and radiotherapy may be beneficial, depending upon when the drug is administered.

Our finding, the benefit of statin therapy particularly after diagnosis, is consistent with several large previous studies. With radio-sensitizing properties, statins have antitumor effects which support their beneficial use in prostate cancer. ${ }^{32}$ Raval et $\mathrm{a}^{33}$ performed a meta-analysis that included 34 observational cohort studies and found a $21 \%$ reduction in the risk of biochemical recurrence $(\mathrm{BCR})$ in patients treated with statin and radiation therapy $(P=0.01)$. However, statin use was not associated with BCR in patients who received radical prostatectomy. Raval et al ${ }^{13}$ also found that statin use was associated with a $22 \%$ reduction in the risk of metastasis, and a $24 \%$ reduction in risk of both all-cause and prostate cancer-specific mortality. Yu et al ${ }^{13}$ performed a large cohort study $(\mathrm{N}=11,772)$ in men with newly diagnosed, nonmetastatic prostate cancer using a large population-based electronic database from the UK. They found that, during a mean follow-up of 4.4 years, 3,499 deaths occurred, of which 1,791 were from prostate cancer. Similar to the results reported in the current study, post-diagnostic use of statins was associated with a $24 \%$ decrease in prostate cancer-specific mortality ( $\mathrm{HR}=0.76 ; 95 \% \mathrm{CI}=0.66-0.88)$ and a $14 \%$ decrease in allcause mortality ( $\mathrm{HR}=0.86 ; 95 \% \mathrm{CI}=0.78-0.95$ ). Therefore, post-diagnostic statin use among prostate cancer patient who were not previously on a statin may be beneficial. ${ }^{35}$ However, in contrast to the current study reported here, $\mathrm{Yu}$ et $\mathrm{al}^{34}$ found that the association of statin use and decreased risks of prostate cancer and all-cause mortality was more 
pronounced in patients who were using statins prior to the diagnosis $(\mathrm{HR}=0.55 ; 95 \% \mathrm{CI}=0.41-0.74$; and $\mathrm{HR}=0.66$; $95 \% \mathrm{CI}=0.53-0.81$, respectively). Larsen et $\mathrm{al}^{36}$ found that there was no difference in the effect of post-diagnostic statin use in Danish prostate cancer patients. Taken together, these studies suggested that the duration and/or timing of exposure might be important in statin therapy. ${ }^{35}$

The importance of statin use following the initiation of prostate cancer therapy was also investigated in a study by Harshman et al, ${ }^{37}$ who retrospectively analyzed 926 men who had received ADT for biochemical or metastatic recurrence. They found, after a median follow-up of 5.8 years, that men using statins had longer median time to disease progression (27.4 months) than patients not taking statins (17.4 months) $(P<0.001)$. The benefit of statin therapy was observed both in patients with and without metastasis. Overall, the findings from the current study and the prior studies support the use of statin therapy after the diagnosis of prostate cancer. One important difference between our study and the previous ones is that our study population only included patients with hyperlipidemia.

A meta-analysis of 22 trials that assessed statin vs placebo for the treatment of cardiovascular disease did not find a significant difference in prostate cancer mortality. ${ }^{38}$ However, the follow-up time was much shorter in the randomized control trials than that of the observational studies described above. Liu et al, ${ }^{39}$ in 2017 , reported that the use of statins and metformin were not significantly associated with treatment outcomes in prostate cancer patients, but lower levels of PSA were observed in patients who were given statins or metformin. Several studies have investigated the association of metformin use and mortality following a prostate cancer diagnosis. A population-based study by Margel et $\mathrm{al}^{40}$ included 3,837 patients with a median follow-up of 4.65 years. They found the cumulative duration of metformin treatment after prostate cancer diagnosis was associated with a significant decrease in the risk of prostate cancer-specific and all-cause mortality; adjusted prostate cancer mortality was 0.76 for each additional 6 months of metformin use. The association with all-cause mortality was also significant, but declined over time from 0.76 in the first 6 months to 0.93 between 24 and 30 months of therapy. The large sample size cohort studies (a total of 13 cohort studies encompassing 177,490 individuals) in 2017 suggested that metformin treatment improves survival for patients with prostate cancer. ${ }^{41}$ The study of Bensimon et $\mathrm{al}^{42}$ included 935 patients from the
UK, and found no association of metformin use with overall decreased risk of cancer-specific and all-cause mortality. A recent meta-analysis of all studies that accounted for the diagnosis of diabetes found metformin use was associated with better overall survival and less BCR among prostate cancer patients. ${ }^{43}$ Metformin is also the most common drug used in type II diabetes mellitus. Zingales et $\mathrm{al}^{22}$ suggested that metformin has anticancer activity and could be potentially used in cancer management. Whereas, Ferro et $\mathrm{al}^{44}$ reported that diabetic patients with a combination treatment of metformin and radiation developed increased acute locoregional toxicity, in comparison with diabetic patients receiving an alternate diabetes medication and nondiabetic patients in breast cancer. More investigation should be conducted to determine the safety in use of metformin in combination with radiation therapy. Although our study suggests a benefit of metformin treatment, the inconsistency across studies indicates additional studies are necessary to further investigate the role of metformin in prostate cancer. The current study has several limitations that should be considered while interpreting the results. Due to the restrictions of the NHIRD database, information including patient lifestyle, family history, patient demographics, cancer stage, and clinical laboratory data were not available and could not be evaluated. A previously published study had reported the limitations caused by the accessibility of their database, which may introduce measurement error in the definition of the duration/timing of statin/metformin therapy and in clinical diagnoses. ${ }^{44}$ There were also limitations associated with the statistical analysis. The data we obtained only allowed for the calculation of overall mortality, so we were unable to determine prostate cancer-specific mortality or diseasefree survival. The study did not evaluate the length of statin and/or metformin use on prostate cancer survival. There were no patients with hyperlipidemia and radiotherapy who received surgical castration or ADT in NHIRD 2000-2010, thus the present study could not evaluate these patients.

In conclusion, the present study suggests that statins and metformin use after the diagnosis of prostate cancer may increase survival in patients with hyperlipidemia who received radiotherapy. These findings, along with other studies, support the use of these medications as adjuvant therapy for the treatment of prostate cancer. However, more studies are necessary to further assess their value and also the timing, length of treatment, and patient population who might benefit most from their use. 


\section{Acknowledgment}

This work was supported by Pearl River S\&T Nova Program of Guangzhou (No. 201710010039), National Natural Science Foundation (No. 81602248), Natural Science Foundation of Guangdong Province (No. 2017A030313898), Science and Technology Program of Guangdong Province (No. 2017A020215028), Science and Technology Program of Guangzhou (No. 201707010113), and Basic Service Charge Young Teachers Cultivation Project of Sun Yat-sen University (17ykpy48).

\section{Author contributions}

$\mathrm{Ke} \mathrm{Li:} \mathrm{conception} \mathrm{and} \mathrm{design;} \mathrm{critical} \mathrm{revision} \mathrm{of} \mathrm{the} \mathrm{manu-}$ script; obtaining funding; final approval of the manuscript; Jianguang Qiu: analysis and interpretation of data; critical revision of the manuscript; obtaining funding; $\mathrm{Li} \mathrm{Lu}$ : acquisition of data; drafting of the manuscript; Yunhua Mao: analysis and interpretation of data; critical revision of the manuscript; Hua Zeng: analysis and interpretation of data; drafting of the manuscript; statistical analysis; literature research; Mingkun Chen: acquisition of data; drafting of the manuscript; definition of intellectual content; Caiyong Lai: acquisition of data; drafting of the manuscript; Dejuan Wang: conception and design; critical revision of the manuscript; obtaining funding; final approval of the manuscript; clinical studies; administrative, technical or material support; Jie Si-Tu: conception and design; critical revision of the manuscript; final approval of the manuscript; definition of intellectual content. All authors contributed to data analysis, drafting and revising the article, gave final approval of the version to be published, and agree to be accountable for all aspects of the work.

\section{Disclosure}

The authors report no conflicts of interest in this work.

\section{References}

1. Chiang CJ, Lo WC, Yang YW, You SL, Chen CJ, Lai MS. Incidence and survival of adult cancer patients in Taiwan, 2002-2012. J Formos Med Assoc. 2016;115(12):1076-1088.

2. Hung CF, Yang CK, Ou YC. Urologic cancer in Taiwan. Jpn J Clin Oncol. 2016;46(7):605-609.

3. Pu YS. Prostate cancer in Taiwan: epidemiology and risk factors. Int $J$ Androl. 2000;23 Suppl 2:34-36.

4. Hamilton RJ, Goldberg KC, Platz EA, Freedland SJ. The influence of statin medications on prostate-specific antigen levels. J Natl Cancer Inst. 2008;100(21):1511-1518.

5. Chang SL, Harshman LC, Presti JC. Impact of common medications on serum total prostate-specific antigen levels: analysis of the national health and nutrition examination survey. J Clin Oncol. 2010;28(25) 3951-3957.
6. Cyrus-David MS, Weinberg A, Thompson T, Kadmon D. The effect of statins on serum prostate specific antigen levels in a cohort of airline pilots: a preliminary report. J Urol. 2005;173(6):1923-1925.

7. Mondul AM, Selvin E, De Marzo AM, Freedland SJ, Platz EA. Statin drugs, serum cholesterol, and prostate-specific antigen in the National Health and Nutrition Examination Survey 2001-2004. Cancer Causes Control. 2010;21(5):671-678.

8. Nordström T, Clements M, Karlsson R, Adolfsson J, Grönberg H. The risk of prostate cancer for men on aspirin, statin or antidiabetic medications. Eur J Cancer. 2015;51(6):725-733.

9. Allott EH, Farnan L, Steck SE, et al. Statin use, high cholesterol and prostate cancer progression; results from HCaP-NC. Prostate. 2018;78(11):857-864

10. Chen YA, Lin YJ, Lin CL, et al. Simvastatin therapy for drug repositioning to reduce the risk of prostate cancer mortality in patients with hyperlipidemia. Front Pharmacol. 2018;9:225.

11. Nielsen SF, Nordestgaard BG, Bojesen SE. Statin use and reduced cancer-related mortality. N Engl J Med. 2013;368(6):576-577.

12. Tan P, Wei S, Yang L, et al. The effect of statins on prostate cancer recurrence and mortality after definitive therapy: a systematic review and meta-analysis. Sci Rep. 2016;6:29106.

13. Park HS, Schoenfeld JD, Mailhot RB, et al. Statins and prostate cancer recurrence following radical prostatectomy or radiotherapy: a systematic review and meta-analysis. Ann Oncol. 2013;24(6):1427-1434.

14. Gutt R, Tonlaar N, Kunnavakkam R, Karrison T, Weichselbaum RR, Liauw SL. Statin use and risk of prostate cancer recurrence in men treated with radiation therapy. J Clin Oncol. 2010;28(16):2653-2659.

15. Caon J, Paquette M, Hamm J, Pickles T. Does statin or ASA affect survival when prostate cancer is treated with external beam radiation therapy? Prostate Cancer. 2014;2014(4):1-6.

16. Platz EA, Tangen CM, Goodman PJ, et al. Statin drug use is not associated with prostate cancer risk in men who are regularly screened. J Urol. 2014;192(2):379-384.

17. Freedland SJ, Hamilton RJ, Gerber L, et al. Statin use and risk of prostate cancer and high-grade prostate cancer: results from the reduce study. Prostate Cancer Prostatic Dis. 2013;16(3):254-259.

18. Akinyeke T, Matsumura S, Wang X, et al. Metformin targets c-myc oncogene to prevent prostate cancer. Carcinogenesis. 2013;34(12): 2823-2832.

19. Ben Sahra I, Laurent K, Loubat A, et al. The antidiabetic drug metformin exerts an antitumoral effect in vitro and in vivo through a decrease of cyclin D1 level. Oncogene. 2008;27(25):3576-3586.

20. Lee SY, Song CH, Xie YB, Jung C, Choi HS, Lee K. SMILE upregulated by metformin inhibits the function of androgen receptor in prostate cancer cells. Cancer Lett. 2014;354(2):390-397.

21. Nguyen HG, Yang JC, Kung HJ, et al. Targeting autophagy overcomes enzalutamide resistance in castration-resistant prostate cancer cells and improves therapeutic response in a xenograft model. Oncogene. 2014;33(36):4521-4530.

22. Zingales V, Distefano A, Raffaele M, Zanghi A, Barbagallo I, Vanella L. Metformin: a bridge between diabetes and prostate cancer. Front Oncol. 2017;7:243.

23. Whitburn J, Edwards CM, Sooriakumaran P. Metformin and prostate cancer: a new role for an old drug. Curr Urol Rep. 2017;18(6):46.

24. Mayer MJ, Klotz LH, Venkateswaran V. The effect of metformin use during docetaxel chemotherapy on prostate cancer specific and overall survival of diabetic patients with castration resistant prostate cancer. J Urol. 2017;197(4):1068-1075.

25. Taira AV, Merrick GS, Galbreath RW, Morris M, Butler WM, Adamovich E. Metformin is not associated with improved biochemical free survival or cause-specific survival in men with prostate cancer treated with permanent interstitial brachytherapy. J Contemp Brachytherapy. 2014;6(3):254-261.

26. Coyle C, Cafferty FH, Vale C, Langley RE. Metformin as an adjuvant treatment for cancer: a systematic review and meta-analysis. Ann Oncol. 2016;27(12):2184-2195. 
27. Deng D, Yang Y, Tang X, et al. Association between metformin therapy and incidence, recurrence and mortality of prostate cancer: evidence from a meta-analysis. Diabetes Metab Res Rev. 2015;31(6):595-602.

28. Liu Y, Huang C, Ceng C, Zhan H, Zheng D, Han W. Metformin enhances nitric oxide production and diminishes Rho kinase activity in rats with hyperlipidemia. Lipids Health Dis. 2014;13:115.

29. Cheng SH, Chiang TL. The effect of universal health insurance on health care utilization in Taiwan. Results from a natural experiment. JAMA. 1997;278(2):89-93.

30. Chiang CL, Chen PC, Huang LY, et al. Impact of universal health coverage on urban-rural inequity in psychiatric service utilisation for patients with first admission for psychosis: a 10-year nationwide population-based study in Taiwan. BMJ Open. 2016;6(3):e010802.

31. Shen CC, Hu LY, Hu YH. Comorbidity study of borderline personality disorder: applying association rule mining to the Taiwan National health insurance Research Database. BMC Med Inform Decis Mak. 2017;17(1):8.

32. Hutchinson J, Marignol L. Clinical potential of statins in prostate cancer radiation therapy. Anticancer Res. 2017;37(10):5363-5372.

33. Raval AD, Thakker D, Negi H, Vyas A, Kaur H, Salkini MW. Association between statins and clinical outcomes among men with prostate cancer: a systematic review and meta-analysis. Prostate Cancer Prostatic Dis. 2016;19(2):151-162.

34. Yu O, Eberg M, Benayoun S, et al. Use of statins and the risk of death in patients with prostate cancer. J Clin Oncol. 2014;32(1):5-11.

35. Mucci LA, Kantoff PW. Is the evidence sufficient to recommend statins for all men with prostate cancer? J Clin Oncol. 2017;35(29):3272-3274.

36. Larsen SB, Dehlendorff C, Skriver C, et al. Postdiagnosis statin use and mortality in Danish patients with prostate cancer. J Clin Oncol. 2017;35(29):3290-3297.
37. Harshman LC, Wang X, Nakabayashi M, et al. Statin use at the time of initiation of androgen deprivation therapy and time to progression in patients with hormone-sensitive prostate cancer. JAMA Oncol. 2015;1(4): 495-504.

38. Cholesterol Treatment Trialists' (CTT) Collaboration, Emberson JR, Kearney PM, et al. Lack of effect of lowering LDL cholesterol on cancer: meta-analysis of individual data from 175,000 people in 27 randomised trials of statin therapy. PLoS One. 2012;7(1):e29849.

39. Liu X, Li J, Schild SE, et al. Statins and metformin use is associated with lower PSA levels in prostate cancer patients presenting for radiation therapy. J Cancer Ther. 2017;8(2):73-85.

40. Margel D, Urbach DR, Lipscombe LL, et al. Metformin use and allcause and prostate cancer-specific mortality among men with diabetes. J Clin Oncol. 2013;31(25):3069-3075.

41. Xiao Y, Zheng L, Mei Z, et al. The impact of metformin use on survival in prostate cancer: a systematic review and meta-analysis. Oncotarget. 2017;8(59):100449-100458.

42. Bensimon L, Yin H, Suissa S, Pollak MN, Azoulay L. The use of metformin in patients with prostate cancer and the risk of death. Cancer Epidemiol Biomarkers Prev. 2014;23(10):2111-2118.

43. Stopsack KH, Ziehr DR, Rider JR, Giovannucci EL. Metformin and prostate cancer mortality: a meta-analysis. Cancer Causes Control. 2016;27(1):105-113.

44. Ferro A, Goyal S, Kim S, et al. Evaluation of diabetic patients with breast cancer treated with metformin during adjuvant radiotherapy. Int J Breast Cancer. 2013;2013:Article ID 659723. 


\section{Supplementary materials}

Table SI Comparison of the difference between characteristics and medicine use

\begin{tabular}{|c|c|c|c|c|c|}
\hline \multirow[t]{2}{*}{ Variable } & \multicolumn{4}{|c|}{ Medicine use } & \multirow[t]{2}{*}{$P$-value } \\
\hline & $\begin{array}{l}\text { Unused } \\
(n=213)\end{array}$ & $\begin{array}{l}\text { Both } \\
(n=|2|)\end{array}$ & $\begin{array}{l}\text { Only use statin } \\
(n=174)\end{array}$ & $\begin{array}{l}\text { Only use metformin } \\
(n=59)\end{array}$ & \\
\hline Age (years) & $71.6 \pm 9.4$ & $72.4 \pm 8.2$ & $70.8 \pm 8.4$ & $74.0 \pm 8.1$ & 0.083 \\
\hline \multicolumn{6}{|l|}{ Comorbidities } \\
\hline Diabetes mellitus & $92(43.2)$ & $102(84.3)$ & $59(33.9)$ & $52(88.1)$ & $<0.001^{a}$ \\
\hline Hypertension & $152(7 \mid .4)$ & $102(84.3)$ & $138(79.3)$ & $45(76.3)$ & $0.046^{\mathrm{a}}$ \\
\hline Cardiovascular & $132(62.0)$ & $86(71.1)$ & $127(73.0)$ & $31(52.5)$ & $0.009^{a}$ \\
\hline \multicolumn{6}{|l|}{ Disease } \\
\hline Peripheral artery & $12(5.6)$ & II (9.1) & I3 (7.5) & $5(8.5)$ & 0.663 \\
\hline \multicolumn{6}{|l|}{ Disease } \\
\hline Atherosclerosis & $17(8.0)$ & $9(7.4)$ & $18(10.3)$ & 7 (II.9) & 0.658 \\
\hline Mortality & $60(28.2)$ & $26(21.5)$ & $35(20.1)$ & $16(27.1)$ & 0.243 \\
\hline
\end{tabular}

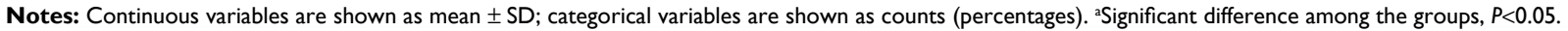

Table S2 Survival analysis of medicine use for patients with diabetes

\begin{tabular}{|l|l|l|l|l|l|l|}
\hline & $\begin{array}{l}\text { n of } \\
\text { events }^{\mathbf{a}}\end{array}$ & $\begin{array}{l}\text { Mean for survival } \\
\text { time (years) }\end{array}$ & $\mathbf{S E}$ & $\begin{array}{l}\text { Log-rank test, } \\
\boldsymbol{P} \text {-value }\end{array}$ & $\begin{array}{l}\text { HR } \\
\mathbf{( 9 5 \% ~ C l )}\end{array}$ & $\begin{array}{l}\text { aHR } \\
\mathbf{( 9 5 \% ~ C I )}\end{array}$ \\
\hline Medicine use & 75 & 7.4 & 0.2 & 0.906 & & \\
$\quad$ Unused & 21 & 7.5 & 0.4 & & $\mathrm{I}$ & $\mathrm{I}$ \\
Both & 25 & 7.3 & 0.4 & & $1.17(0.65-2.08)$ & $1.32(0.73-3.06)$ \\
Only use statin & 15 & 7.2 & 0.6 & & $1.22(0.63-2.37)$ & $1.53(0.76-3.06)$ \\
Only use metformin & 14 & 6.9 & 0.5 & & $1.25(0.64-2.46)$ & $1.43(0.72-2.83)$ \\
\hline
\end{tabular}

Notes: ${ }^{\text {In }}$ survival analysis, any cause of death was treated as an event. The Multivariate Cox proportional hazards regression model was simultaneously adjusted for age, hypertension, cardiovascular disease, peripheral artery disease, and atherosclerosis.

Abbreviations: aHR, adjusted hazard ratio, SE, standard error.

Table S3 Survival analysis of medicine use in only use statin or metformin group for patients with diabetes

\begin{tabular}{|c|c|c|c|c|c|c|}
\hline & $\begin{array}{l}n \text { of } \\
\text { event }\end{array}$ & $\begin{array}{l}\text { Average survival } \\
\text { time (years) }\end{array}$ & SE & $\begin{array}{l}\text { Log-rank test, } \\
P \text {-value }\end{array}$ & HR $(95 \% \mathrm{Cl})$ & aHR $(95 \% \mathrm{Cl})$ \\
\hline Only use statin & & & & $0.006^{b}$ & & \\
\hline Unused & 21 & 7.5 & 4.1 & & I & I \\
\hline Persistent use & 8 & 6.2 & 0.8 & & I.7I (0.42-3.88) & $2.57(1.03-6.45)$ \\
\hline Pre-diagnostic use & 7 & 4.5 & 0.6 & & 2.71 (1.13-6.49) & $2.96(I .18-7.4 I)$ \\
\hline Post-diagnostic use & 0 & - & - & & - & - \\
\hline Only use metformin & & & & $<0.00 I^{\mathrm{b}}$ & & \\
\hline Unused & 21 & 7.5 & 0.4 & & I & I \\
\hline Persistent use & 8 & 7.1 & 0.6 & & $1.19(0.52-2.69)$ & $1.39(0.60-3.24)$ \\
\hline Pre-diagnostic use & 5 & 1.9 & 0.5 & & $7.06(2.54-19.59)$ & $7.59(2.58-22.33)$ \\
\hline Post-diagnostic use & 1 & 7.9 & 0.4 & & $0.28(0.04-2.07)$ & $0.38(0.05-2.87)$ \\
\hline
\end{tabular}

Notes: aln survival analysis, any cause of death was treated as an event. ${ }^{b}$ Represented a significant difference of average survival time among the groups, log-rank test, $P<0.05$. Bold represented a significant factor, $P<0.05$; the multivariate Cox proportional hazards regression model was simultaneously adjusted for age, hypertension, cardiovascular disease, peripheral artery disease, and atherosclerosis.

Abbreviation: aHR, adjusted hazard ratio, HR, hazard ratio; SE, standard error.

Cancer Management and Research

\section{Publish your work in this journal}

Cancer Management and Research is an international, peer-reviewed open access journal focusing on cancer research and the optimal use of preventative and integrated treatment interventions to achieve improved outcomes, enhanced survival and quality of life for the cancer patient. The manuscript management system is completely online and includes

Dovepress

a very quick and fair peer-review system, which is all easy to use. Visit $\mathrm{http}: / / \mathrm{www}$.dovepress.com/testimonials.php to read real quotes from published authors.

Submit your manuscript here: https://www.dovepress.com/cancer-management-and-research-journal 